364-369.

DANIN, A. in GREUTER W, \& T.H. RAUS, Eds. -2006- Med-Checklist Notulae, 24. Arundo mediterranea Danin. Willdenowia 36: 727.

DANIN, A. in GREUTER W, \& T.H. RAUS, Eds. -2007- Med-Checklist Notulae, 25. Arundo mediterranea Danin. Willdenowia 37: 213.

DANIN, A., T.H. RAUS, \& H. SCHOLZ -2002Contribution to the flora of Greece: a new species of Arundo (Poaceae). Willdenowia 32: 191-194.

DANIN, A. \& G. HADJIKYRIAKOU in GREUTER W, \& T.H. RAUS, Eds. -2004- Med-Checklist Notulae, 22. Arundo plinii Turra. Willdenowia 34: 78.

DANIN, A., G. DOMINA \& F.M. RAIMONDO -2005- Prime osservazioni ecologico-distributive su Arundo collina Ten. (Poaceae) in Italia. Inform. Bot. Ital. 37(2): 1167-1170.
DANIN, A. \& W. NAENNY -2008- Contribution to the recognition of reeds by their rhizome anatomy. Fl. Medit. 18: 385-392.

DANIN, A., C. BLANCHÉ, F. ROYO \& M. ARRUFAT in GREUTER W, \& T.H. RAUS, Eds. -2008- Med-Checklist Notulae, 27. Arundo mediterranea Danin. Willdenowia 38(2): 469.

DESFONTAINES, R. -1798-99- Flora atlantica 1-2. Paris.

Dirección de los autores: ${ }^{1}$ Centro Conservazione Biodiversità (CCB), Dipartimento di Scienze della Vita e dell'Ambiente, Università degli Studi di Cagliari. Viale S. Ignazio da Laconi 13, 09123 Cagliari (Italia); ${ }^{2}$ Grupo de Sistemas y Recursos Forestales, Área de Recursos Naturales, IFAPA Centro Camino de Purchil. Camino de Purchil s/n. Aptdo. 2027. 18080 Granada (España).

*Autor para correspondencia: fbruno.navarro@ juntadeandalucia.es

\title{
217. ERIGERON ATTICUS VILL. (ASTERACEAE) EN EL NORESTE DE LA PENÍNSULA
} IBÉRICA

\author{
Antonio J. PUJADAS SALVÀ ${ }^{*}$ y Vicente J. ARÁN REDÓ²
}

Recibido el 17 de junio de 2011, aceptado para su publicación el 1 de julio de 2011

Erigeron atticus Vill. in north-eastern Iberian Peninsula

Palabras clave. Erigeron, corología, Andorra, Lleida.

Key words. Erigeron, chorology, Andorra, Lleida.

E. atticus [atticum] Vill., Hist. Pl. Dauphiné 3(1): 237 (1789)

$\equiv$ E. alpinus var. atticus (Vill.) Fiori, Fl. Anal.
Ital. 3: 234 (1903)

三 Trimorpha attica (Vill.) Vierh., Beih. Bot. Centralbl. 19 (2): 461 (1906) 


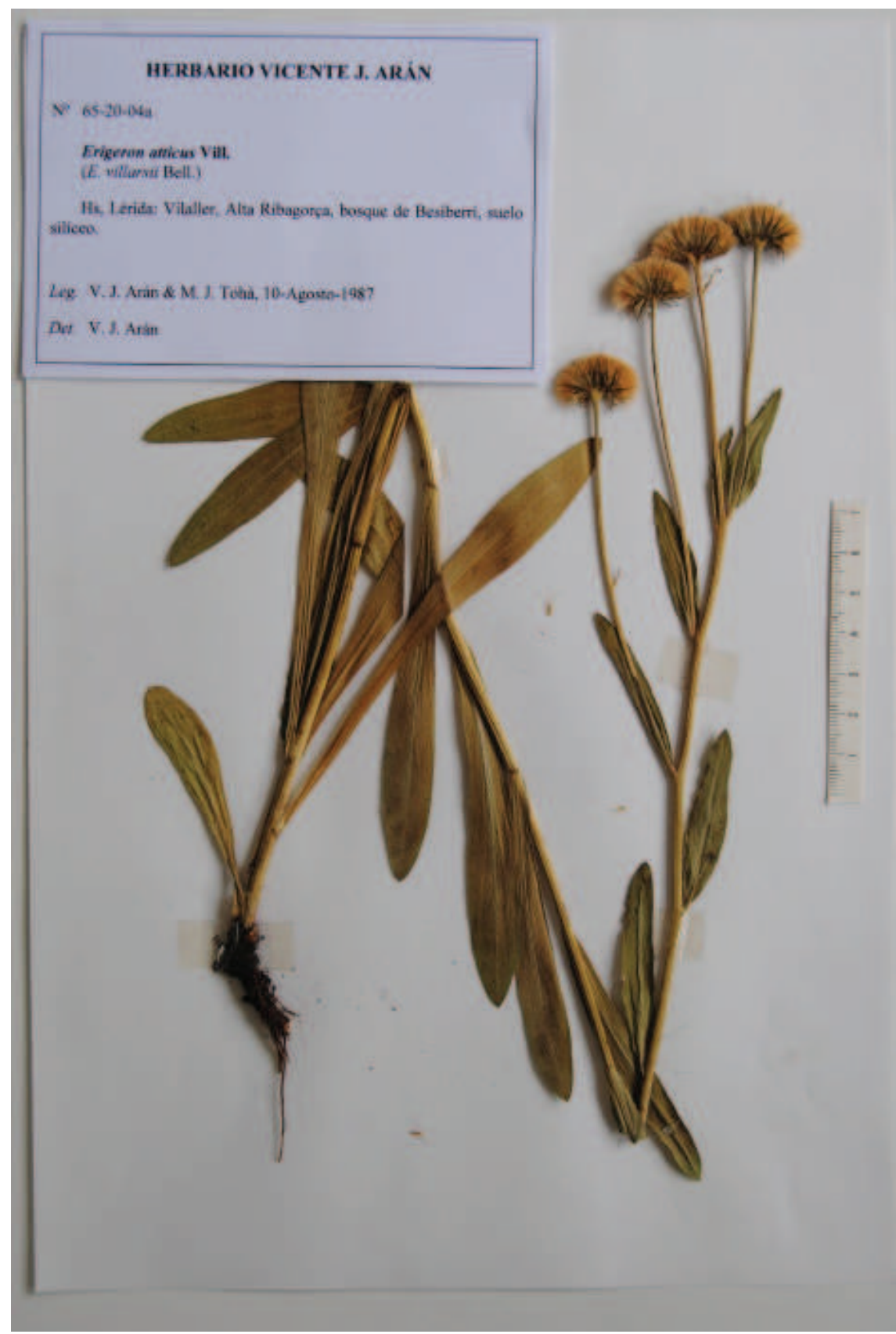

Figura. 1. Erigeron atticus Vill., Lleida, Vilaller, Alta Ribagorça, bosque de Besiberri, $\left(\mathrm{H}^{\circ}\right.$ V.J. ARÁN 65-20-04a). Erigeron atticus Vill., Lleida, Vilaller, Alta Ribagorça, bosque de Besiberri, (H ${ }^{\circ}$ V.J. ARÁN $65-20-04 a)$ 
$=$ E. villarsii Bellardi, App. Fl. Pedem. 38. t. 7 (1792) (nom. illeg.)

Material estudiado. ANDORRA: Montagnes au dessus du Col de la Botella, 10-VIII-1980, J. Bouchard 335, $\mathrm{H}^{\circ}$ BOUCHARD. CIENMA-IEA (sub E. villarsii). Parroquia de Canillo, riu Aixec, $42^{\circ} 36^{\prime} 07^{\prime \prime} \mathrm{N}$ $01^{\circ} 40^{\prime} 25^{\prime \prime}$ E, 1790 m, 26-VII-2005, C. Aedo, I. Aizpuru \& J. Pedrol 12146, MA 732173. ESPAÑA. Lleida: Vilaller, Alta Ribagorça, bosque de Besiberri, 10-VIII-1987, V.J. Arán \& M.J. Tohá, H V.J. ARÁN 65-20-04a (Fig. 1). Val d'Aran, Colomers, lac de Clòto, 31TCH32, 2200 m, 25-VI-2003, I. Aizpuru, ARAN 65719.

Erigeron atticus Vill. es una especie propia de herbazales megafórbicos, en terrenos pedregosos de suelos ácidos, distribuida por las montañas del centro y sur de Europa, Alpes, Cárpatos y Balcanes, y que alcanza por el oeste los Pirineos Orientales. Es una hierba perenne, rizomatosa, por lo general unicaule, con tallo simple, hasta de $65(75) \mathrm{cm}$, cubierta de pelos cortos glandulíferos y pelos eglandulosos largos esparcidos. Presenta (4)5-9 capítulos, de flores trimórficas (con flores filiformes entre las flores hemiliguladas de la periferia y las flores flosculosas centrales), agrupados en conflorescencias racemiformes -a veces paniculiformes- de aspecto \pm corimboso, con involucro de (15)18-35 mm de diámetro y lígulas muy sobresalientes (fig. 1).

La primera cita conocida de los Pirineos se debe a Gautier (1897: 240, sub E. villarsii), quien la indica del "vallée d'Eyne [Eina], rochers rive droite à $2100 \mathrm{~m}$, et au roc de l'Aigle (¿Castellet de Py; Herb. Xatart)”, en la Cerdaña francesa. Su presencia en los Pirineos franceses fue nuevamente confirmada por Braun-Blanquet (1945: 233) para el Canigou, y más recientemente por Altimira i Noguero (2011) también para el valle de Eina, bajo el Puigmal.

$\mathrm{Su}$ presencia en territorio ibérico había sido cuestionada por Losa \& Montserrat (1951:
99), quienes señalaron que “.... los E. neglectus Kerner y E. villarsii Bell., que Braun-Blanquet da como encontrados en el Pirineo Oriental francés, no los hemos visto en Andorra". Sin embargo, es precisamente de aquí de donde se conoce la única referencia sobre la especie en el territorio de Flora iberica, debida a Bouchard (1981: 65, sub E. villarsii), quien la indica para el Coll de la Botella, una cita avalada por el testimonio de herbario que se conserva en el Institut d'Estudis Andorrans-CENMA y que próximamente será incluido en el Herbario virtual del CENMA (2011) (Caritg, com. pers.). Autores posteriores, como Bolòs \& Vigo (1996: 714), Carrillo et al. (2008: 105, 470, 449) y Font i Castell \& Vigo i Bonada (2007: 3541), se limitan tan solo a recoger las citas anteriores.

Se trata de una especie rara en el territorio peninsular, cuya presencia ha sido confirmada nuevamente tras la reciente revisión del género para Flora iberica, así como ampliada su área de distribución peninsular a la provincia de Lleida.

AGRADECIMIENTOS. A los responsables de los herbarios ARAN y MA por las facilidades en la consulta del material. A Llorenç Sáez Gonyalons por la información bibliográfica. A Roger Caritg (IEA) por facilitarnos el pliego de Bouchard. A Joan Altimira i Noguero por la información corológica.

\section{BIBLIOGRAFÍA}

ALTIMIRA I NOGUERO, J. -2011- Flors silvestres de Catalunya. Publicado en Internet http://www. florssilvestresdecatalunya.com/presentacio flors_silvestres_de_catalunya.php [accesión el 30 de Mayo de 2011].

BOLÒS, O. \& J. VIGO -1996- Flora dels Països Catalans, vol. 3. Barcino. Barcelona.

BOUCHARD, J. -1981- Primer herbari de la Flora d'Andorra. Institut d'Estudis Andorrans, Centre de Perpinyà. Perpiñán.

BRAUN-BLANQUET, J. -1945- Notes critiques sur la flore des Pyrénées Orientales. Trav. Soc. Pharm. Montpellier 4: 219-236. 
CARRILLO, A., A. MERCADÉ, J.M. NINOT, J. CARRERAS, A. FERRÉ \& X. FONT -2008Check-list i llista vermella de la flora d'Andorra. Centre d'Estudis de la Neu i de la Muntanya d'Andorra (CENMA) de l'Institut d'Estudis Andorrans (IEA). Andorra.

CENMA -2011- Herbari virtual d'Andorra. Centre d'Estudis de la Neu i la Muntanya d'Andorra. Publicado en Internet http://www. iea.ad [accesión el 15 de Junio de 2011].

FONT I CASTELL, X. \& J. VIGO I BONADA

-2007-Atlas corologic de la flora vascular dels Països Catalans 14. ORCA, Institut d'Estudis Catalans. Barcelona.
GAUTIER, G. -1897- Catalogue raisonné de la flore des Pyrénées-Orientales. [Cat. Fl. PyrénéesOrientales] Société agricole, scientifique et littéraire des Pyrénées-Orientales. Perpignan.

LOSA, T.M. \& P. MONTSERRAT.-1951-Aportación al conocimiento de la Flora de Andorra. Instituto de Estudios Pirenaicos, CSIC. Zaragoza.

Dirección de los autores. ${ }^{1}$ Dpto. Ciencias y Recursos Agrícolas y Forestales, Edificio Celestino Mutis, Campus de Rabanales, Universidad de Córdoba, E-14071 Córdoba y Jardín Botánico de Córdoba, Avda. de Linneo s/n, E-14004 Córdoba. ${ }^{2}$ Instituto de Química Médica (CSIC), Juan de la Cierva 3, E-28006 Madrid. *Autor para correspondencia: cr1pusaa@uco.es

218. ¿QUÉ ES ERIGERON VERGUINII SENNEN (ASTERACEAE)?

Antonio J. PUJADAS SALVÀ

Recibido el 21 de junio de 2011, aceptado pata su publicación el 1 de julio de 2011

What is Erigeron verguinii Sennen (Asteraceae)?

Palabras clave. Erigeron, Erigeron atticus, tipo.

Key words. Erigeron, Erigeron atticus, typus.

Erigeron verguinii fue descrito por Sennen (1936: 19) con material pirenaico procedente de la Cerdaña francesa ["Cerdagne: Gorges de Llo, éboulis calcaires herbeux de Castellvidre, vers 1520 m"], y asimilado con dudas al complejo de E. villarsii Bellardi. Con posterioridad, la especie no ha sido reconocida ni citada como sinónimo por los diferentes autores que han estudiado la flora francesa o del NE de España, como Jovet \& Vilmorin (1975), Halliday (1976), Fournier (1977), Guinochet \& Vilmorin (1982), Bolós \& Vigo (1996), Font i Castell \& Vigo i Bonada (2007).

Se trata, efectivamente, de un taxón poco CLI).

Trabajo financiado parcialmente por el Ministerio de Ciencia e Investigación (CGL 2008-02982-C03-03/ 